\title{
COLONIZACIÓN Y SUCESIÓN DE NUEVOS MEDIOS ACUÁTICOS 11. VARIACIÓN TEMPORAL DE LA COMPOSICIÓN Y ESTRUCTURA DE LAS COMUNIDADES DE INSECTOS
}

\author{
J. Velasco, A. Millán, L. Rarnírez-Díaz. \\ Departamento de Biología Animal y Ecología, Facultad de Biología, Universidad de Murcia. Campus Universitario de Espinardo, \\ 30100 Murcia, Spain.
}

Palabras clave: Colonización, sucesión primaria, insectos acuáticos, comunidad, estructura.

\author{
ABSTRACT \\ COLONIZATION AND SUCCESSION OF NEW AQUATIC SYSTEMS \\ II. TEMPORAL VARIATION OF THE COMPOSITION AND STRUCTURE OF INSECTS COMMUNITIES
}

In this second paper, temporal changes in composition and structure of insect communities in each pond were analyzed in order to establish the patterns of colonization and succession and to determine the most important differences in relation to environmental conditions of studied ponds. Colonization sequence showed a clear seasonal pattem, that was determined principally by available food resources in ponds. Culiseta longeareolata and Chironomus riparius, two species detritivorous, were the first colonists. In spring, colonized other detritivorous and herbivorous species. As increase complexity of communities, amved the first camivorous species, like as Procladius sagittalis. Odonata and Notonectidae species, the large predators, colonized in summer.

Among environmental factors studied, salinity and temporality are the most important in determine changes on colonization and succession processes. Another factors, although did not change the general pattern, affected the establishment and abundance of certain populations, like as the substrate composition on tubicoles Chironomids; and the presence of aquatic macrophytes on mosquitoes populations.

\section{INTRODUCCIÓN}

Uno de los principales problemas en cualquier estudio de sucesión es la separación entre factores abióticos y bióticos e identificar su modo de acción. TANSLEY (1929) divide las causas de sucesión en dos categorías: alogénicas y autogénicas. Las fuerzas alogénicas son externas al sistema e incluyen los ciclos estacionales, climáticos y geomorfológicos, mientras que los cambios autogénicos son inducidos por la biota (ej. competencia, predación), aunque en realidad ambos tipos de causas se superponen.

CONNELL y SLATYER (1977) separan a su vez, los elementos autogénicos en componentes interactivos y no-interactivos. Según la hipótesis no-interactiva, la sucesión es consecuencia de las características individuales de la historia de vida de la biota. La hipótesis alternativa de tipo interactivo, es que las comunidades predecesoras pueden tanto inhibir como facilitar la ascensión de sus sucesoras.

Limnética,9: 87-98 (1993)

(C) Asociación Española de Limnología, Madrid.Spain
La sucesión primaria está inicialmente bajo el control de la colonización. La colonización inicial de un medio es en si misma bastante variable y puede afectar la subsecuente dominancia de las especies. La estructura temprana de la comunidad parece estar determinada por las condiciones abióticas reinantes durante la colonización inicial, mientras que los cambios subsecuentes en la estructura de la comunidad parecen estar fuertemente influenciados por las interacciones entre los organismos (FISHER, 1983). Tolerancia, facilitación e inhibición son tres clases de mecanismos que pueden explicar la secuencia sucesional (CONNELL \& SLATYER, 1977; CONNELL, NOBLE \& SLATYER, 1987). En cualquier caso, una especie sólo se presentará donde y cuando existan las condiciones y los recursos apropiados, y sus competidores y depredadores no la eliminen.

En este segundo articulo, se analizan los cambios sucesionales de las comunidades de insectos en los diferentes estanques durante el primer año de su existencia, en términos de compo- 
sición, abundancia relativa y diversidad, estableciéndose la secuencia de colonización y pattern sucesional, y determinando las diferencias más importantes en relación a las características ambientales iniciales de los medios estudiados.

\section{MATERIAL Y MÉTODOS}

La utilización de 10 pequeños estanques de igual tamaño pero diferentes respecto al régimen hídrico, época de llenado, grado de insolación, tipo de sustrato, presencia y tipo de vegetación, y concentración de nutrientes y sales del agua, según las condiciones a las que fueron sometidos inicialmente, ha permitido caracterizar y cuantificar los procesos de colonización y sucesión por insectos acuáticos que tienen lugar en estos medios durante el primer año de su existencia. En el primero de los dos artículos presentados (VELASCO et al., en este volumen), aparece explicado con detalle el diseño experimental de los estanques y los métodos empleados en el estudio.

Para poder interpretar los cambios temporales en la composición y estructura de la comunidad se han analizado, en base a la variación temporal de cada una de las poblaciones componentes (VELASCO, 1989), la secuencia de colonización de las especies de insectos acuáticos en cada uno de los estanques y los cambios en el tiempo de las abundancias relativas de los diferentes componentes de la comunidad y de la diversidad.

\section{RESULTADOS}

\section{Secuencia de colonización}

En la Fig. 1 se representa la secuencia de aparición de especies y el tiempo de permanencia de estas, en cada uno de los estanques, durante el periodo de estudio. Se ha elaborado teniendo en cuenta la presencia de cualquiera de los estados del ciclo de vida, considerando que una especie está presente en el intervalo entre dos fechas de muestreo donde ha sido registrada, siempre que este no sea superior a un mes.

El tiempo de permanencia de las especies varía dependiendo fundamentalmente, de su reproducción en los estanques, del número de generaciones que realizan y de las interacciones que se producen entre ellas.

La colonización de los estanques I, III, V, VI, VII, VIII y IX es muy rápida, estableciéndose las especies durante el primer o segundo mes, después de su llenado. En cambio, los estanques IV, II y X son colonizados más tarde, en Febrero, Marzo y Junio, respectivamente. En el estanque X, es evidente que la alta salinidad de sus aguas limita la colonización y asentamiento de las especies (VELASCO $\boldsymbol{e t} \boldsymbol{a l}$. , en este volumen).
En general, todos los estanques siguen la misma pauta de colonización, salvo el VIII y el X, que se comentarán más adelante. Al igual que en otros medios acuáticos de nueva creación los Dípteros son los primeros colonizadores (MILNER, 1987; MALMQVIST, 1991). Culiseta longeareolata y Chironomus riparius, especies típicamente oportunistas de escasos requerimientos alimenticios, son, normalmente, las que primero se establecen y componen la comunidad básica de los estanques después de ser llenados. En Enero no se registra la aparición de nuevas especies, debido a las rigurosas condiciones climáticas de este mes, donde se alcanzan las temperaturas mínimas más bajas $\left(-0,5^{\circ} \mathrm{C}\right)$. Sin embargo, a partir de Febrero, cuando las temperaturas empiezan a aumentar y desarrollarse las comunidades fitoplanctónicas, van apareciendo otros Dípteros detritívoros como Dasyhelea sp.1 y Tipula sp., y herbívoros como los Orthocladinos Cricotopus sylvestris, Cricotopus sp.2, Psectrocladius barbimanus y Psectrocladius limbatellus. Procladius sagittalis, la única especie carnívora de los Quironómidos registrados, aparece un poco más tarde cuando sus requerimientos nutritivos están asegurados. Esta especie se alimenta fundamentalmente de larvas de otros Quironómidos y microinvertebrados (BAKER \& McLACHLAN, 1979).

En Febrero, también coloniza el Efemeróptero Cloeon dipterum, que se mantiene en los estanques, al igual que la especie anterior, durante todo el periodo de estudio. En estas fechas, aparece a su vez, el Coleóptero Haliplus lineatocollis, en el estanque III, que se alimenta exclusivamente de Caráceas (FRANCISCOLO, 1979).

A partir de Mayo, cuando las temperaturas son bastante elevadas y la comunidad de los estanques mucho más compleja, comienzan a asentarse algunas especies de Heterópteros y Coleópteros, como Hydroglyphus pusillus en los estanques IV, V, VI, VII y IX; Heliocorisa vermiculata en los estanques II, VII y IX; y Sigara lateralis en el estanque VI.

Ya en los meses típicamente de verano, se establecen gran número de especies de Quironómidos, principalmente Tanytarsinos, Cladotanytarsus atridorsum, Tanytarsus ejuncidus y Tanytarsus sp.2; y también el Quironomino Polypedilum laetum que sustituye a las poblaciones de Chironomus riparius. Todas estas especies requieren para su desarrollo, la presencia en el medio de abundante sedimento, donde construyen sus tubos y se alimentan de detritos (PINDER, 1986); condiciones que se dan favorablemente durante estos meses. También hace su presencia Dasyhelea sp.2 en los estanques VI, VII y IX; Culex pipiens pipiens en los estanques IV, VI y VII; y Caenis luctuosa en los estanques II, III, IV y V. Por otro lado, colonizan los grandes depredadores, como los Odonatos Ortethrum cancellatum en los estanques III, IV y VII, y Crocothemis 
ESTANQUE /

Chironomus riparius Dasyhelea spt Cricotopus sylvestris Procladius sagittalis Polyped lum pull lum Psectrocladius barbimanus Culiseta Longeareolata Anipsos sardea

HeLochares lividus

Potamonectes mariae Cloeon dipterum cladotanyarsus atridorsum Agabus sp

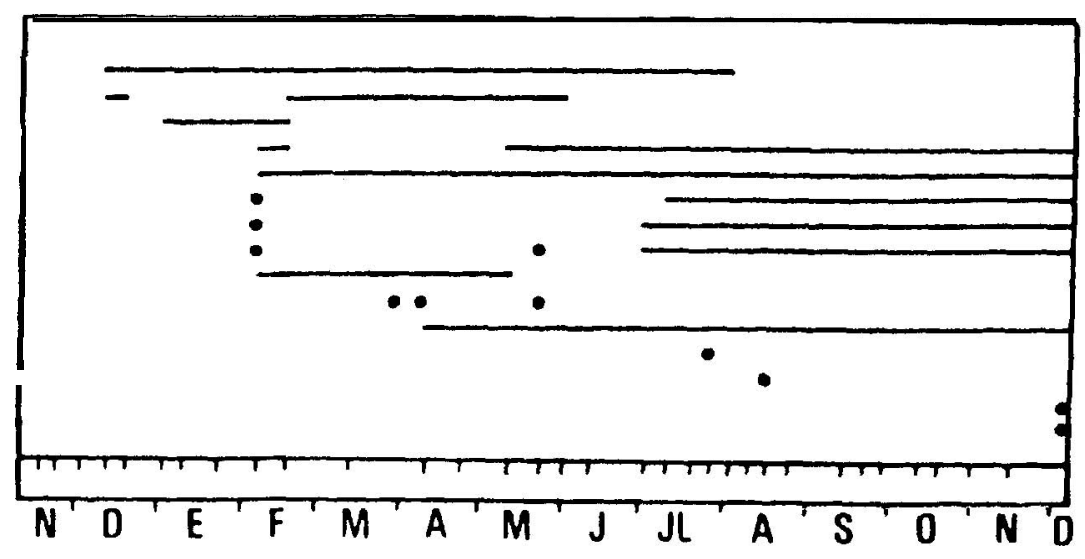

ESTANQUE I I

Ch ironomus riparius Dasyhe Lea spl Cricotopus sp2 procladius sagittalis Tipula sp

Psectrocladius barbimanus Cricotopus sylvestris ochtebius meridional is Tanytarsus ejuncidus Cloeon dipt erum Heliocorisa vermiculata Helochares lividus Polypedilum laetum caenis luctuosa cladotanytarsus atridorsum Tanytarsus sp? Culiseta londeareolata

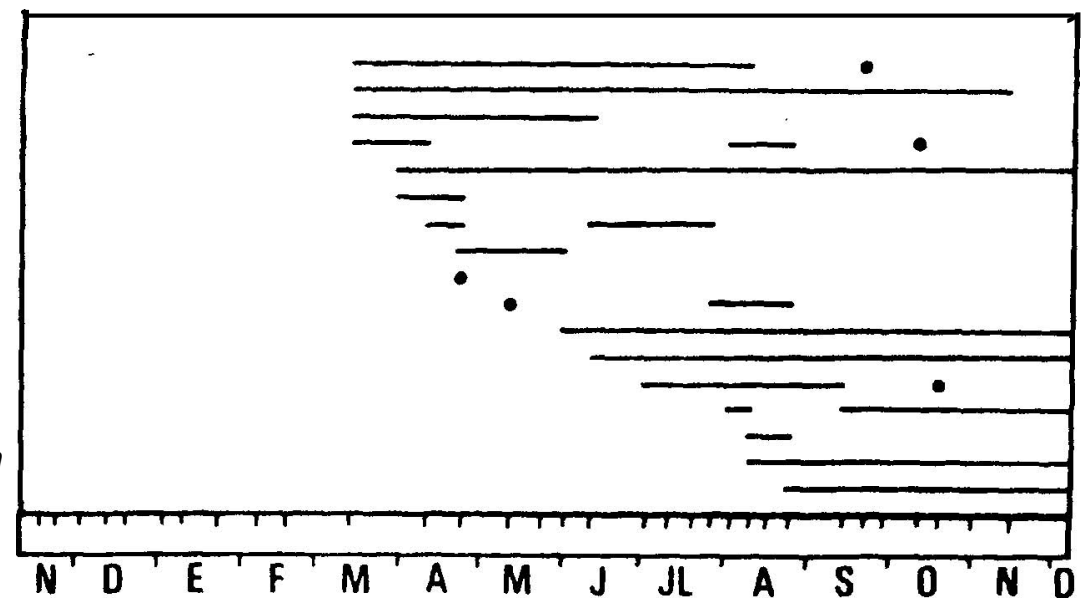

ESTANQUE III

Chironomus riparius Polypedilum laetum Coegn dipterum Haliplus lineatocollis Psectrocladius barbimanus Psectrocladius limbatellus Dasyhelea spl Tanytarsus ejuncidus Culiseta longeareolata procladius sagittalis cricotopus sylvestris Caenis luctuosa cladotanytarsus atridorsum Tanytarsus sp? Orthetrum cancel latum Cricotopus spz Anisops sardea

Heliocorisa vermiculata

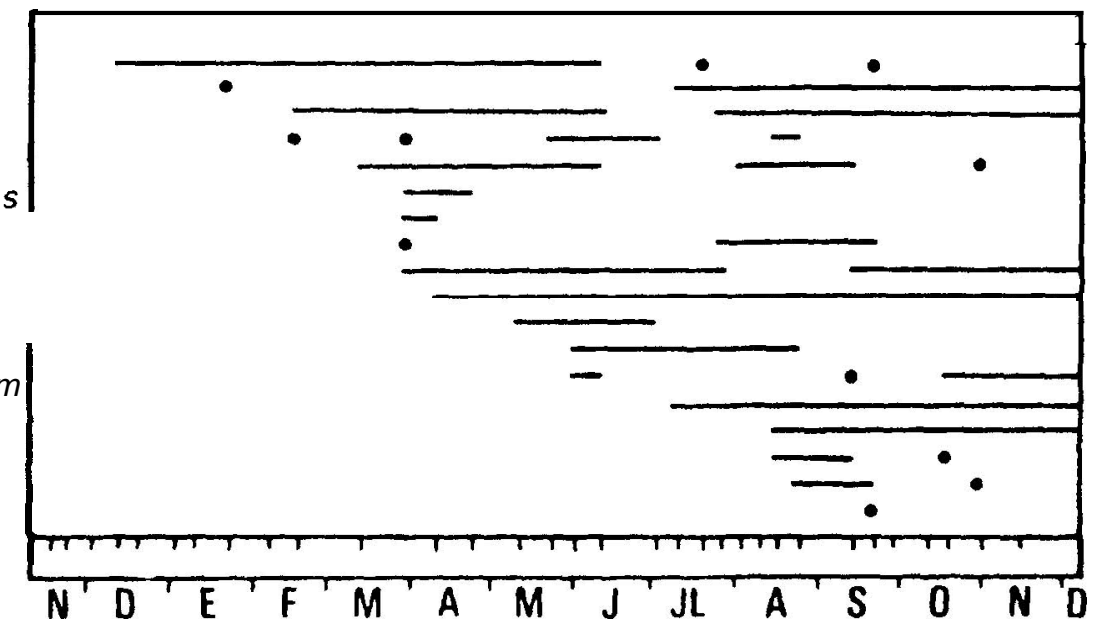

FIGURA 1: Secuencia de colonización y tiempo de residencia de las especies en cada estanque FIGURE 1: Sequence of colonization and residence time of species in each pond 
ESTANQUE IV

Cul iseta Longeareolata Psectrocladiys limbatellus Dasyhe Lea sp1

Cloeon dipterum

Chironomus riparius

Procladius sagittalis

Haliplus lineatoco

Caenis luctuosa

Hydroglyphus pusil Lus

Hel iocorisa vermiculata

Polypedilum laetum

Tanytarsus ejuncidus

Cladotanytarsus atridorsum

Culex pipiens pipiens

Tanytarsus spe

Crocothemis erythraea

Orthetrum cancellatum

Anisops debilis-perplexa

Cricotopus spz

Microvelia pygmaea

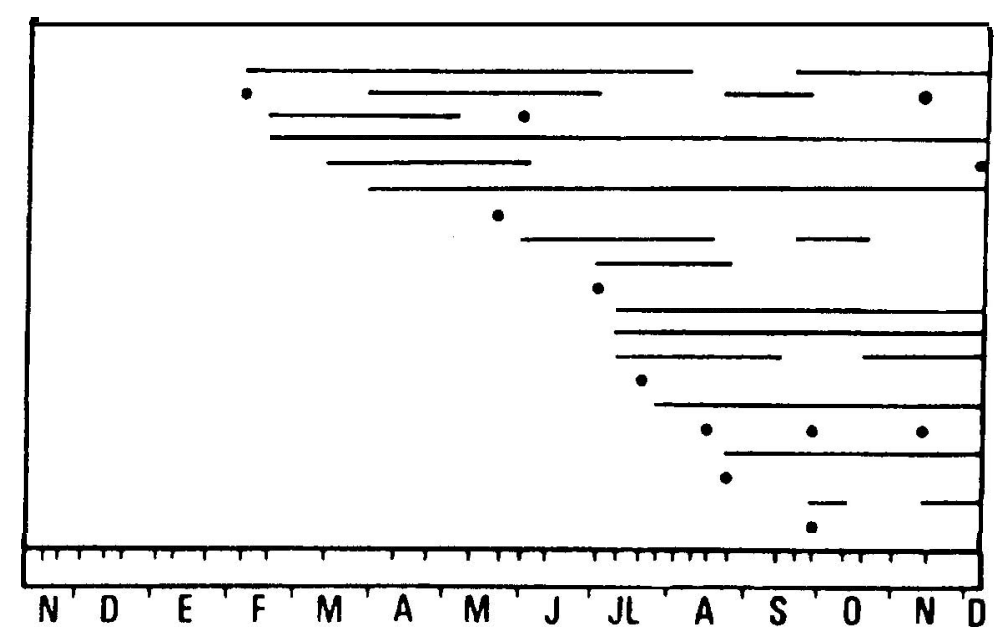

ESTANQUE V

Culiseta longeareolata

Chironomus riparius

Procladius sagittal is

Psectrocladius barbimanus

Cloeon dipterum

Hydroglyphus pus illus

Cricotopus sp?

Tanytarsus ejuncidus

Cladotanytarsus atridorsum

Caenis luctuosa

Microvel ia pygmaea

Helochares lividus

PoLypedilum Laetum

Heliocorisa vermiculata

Anisops debilis-perplexa

Ephydra $s p$

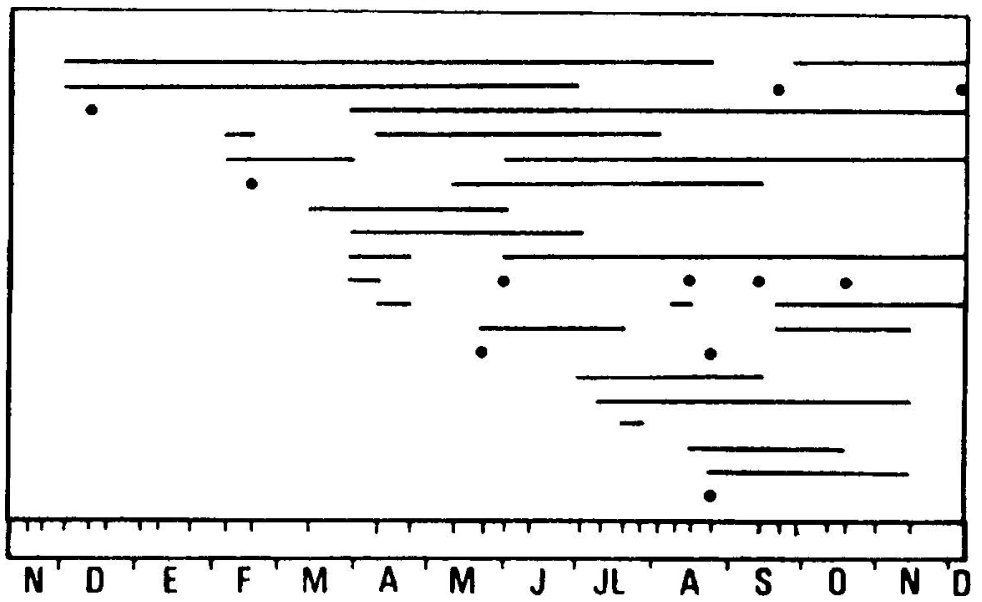

ES TANQUE V I

CyLiseta LonqeareoLata

Chironomus riparius

CLoeon dipterum

Psectrocladius barbimanus

Tanytarsus ejuncidus

Cricotopus sylvestris

Procladius sagittalis

Dasybelea spl

Hyóroglyphus pusillus

Sigara lateralis

Tanytarsus sp

Hel iocorisa vermiculata

Dasyhelea Sp2

culex pipiens pipiens

ochthebius meridional is

ochthebius meridionalis

Cladotanytarsus atr

Anisops debilis-perplexa

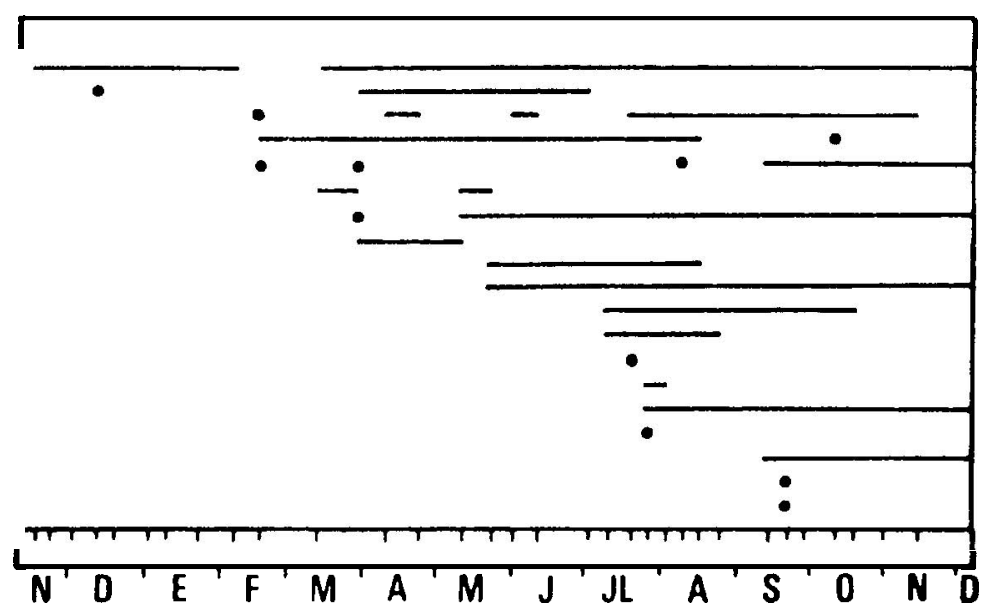

FIGURA 1 (Continuación). 


\section{ESTANQUE VII}

Culiseta lonqeareolata Chironomus riparius

Dasyhelea sp t

Psectrocladius barbimanus

Dasyhelea sp2

Procladius sagittalis

Tanytarsus ejuncidus

Heliocorisa vermiculata

hydrogiyphus pusillus

Tanytarsus spe

cricotopus soylvestris

culex pophens pipiens

Polypedilum Latum

Arthetrum cance

cladotanytarsus atridorsum

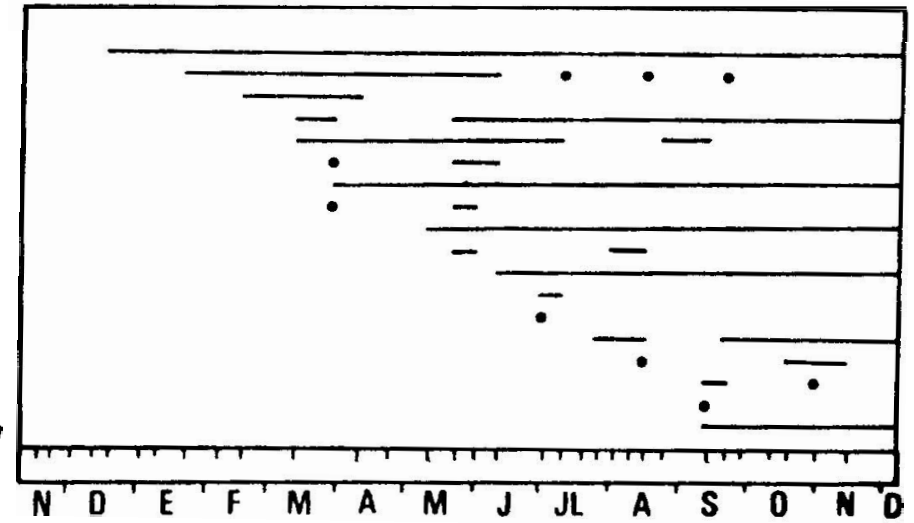

ESTANQUE VIII

Cul iseta longeareolata Chironomus riparlus

Dasyhelea sp?

Dasyhelea sp2

Hel iocor is a vermiculata

Tanytarsus sp?

Polypediltum Laetım

Anisops debilis-perplexa

Procladius sagittalis

Tanytarsus ejuncidus

Psectrocladius barbimanus

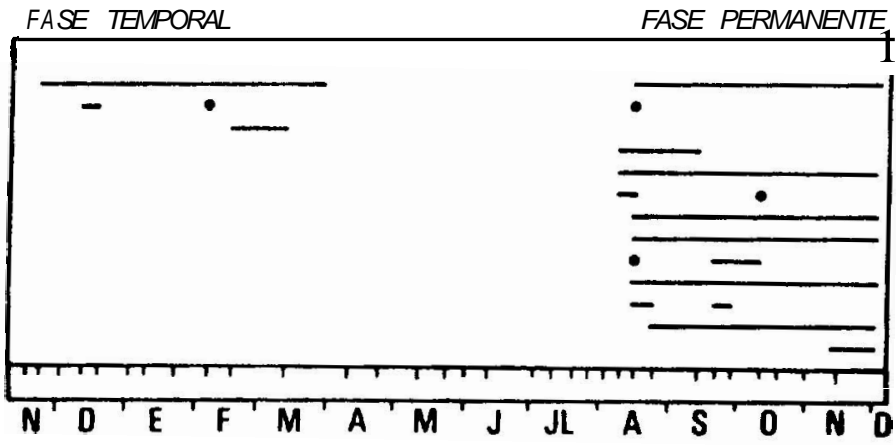

ESTANQUE IX

Culiseta longeareolata Procladius sagit talis

Cloeon dipterum

Chironomus riparius

Dasyhelea sp

Tanytarsus ejuncidus

Psectrocladius barbimanus

heliocorisa vermiculata

Hydroglyphus pusillus

Cricotopus sylvestr

ochthebius meridionalis

Ochthebius mer

Dasyhe lea sp2

any

Helochares lividus

Ephydra so

chironomini spl

Caenis luctuosa

Microvelia pyamaea

Anisops debilis-perplexa

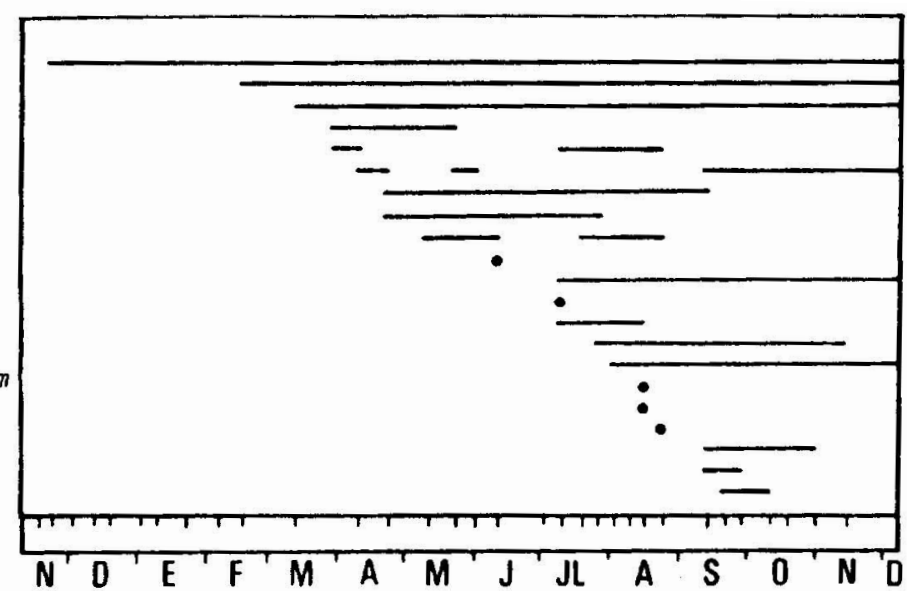

ESTANQUE X

Ephydra $s p$

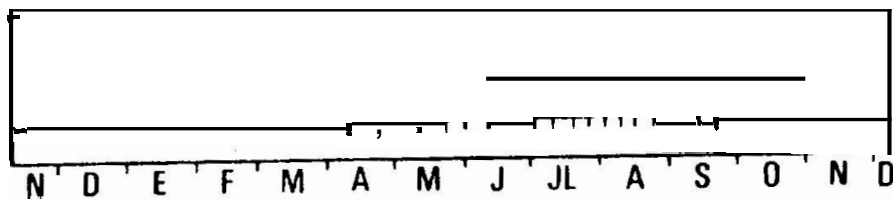

FIGURA 1 (Continuación) 
erythraea en los estanques $\boldsymbol{N}$ y V; el Heteróptero Anisops debilis perplexa en el estanque $\mathrm{V}$, y el Coleóptero Helochares lividus en los estanques II y $\boldsymbol{V}$.

Es en estos meses de verano, cuando se observa en la mayoría de los estanques, la aparición de adultos aislados de algunas especies de Coleópteros y Heterópteros, que utilizan estos medios como lugares de alimentación en sus vuelos de dispersión.

En Otoño, no coloniza ninguna especie nueva, salvo en el estanque I, donde se registran, a principios de Diciembre, los coleópteros Potamonectes mariae y Agabus sp., ambos carnívoros.

En el estanque VIII, se producen dos procesos de colonización totalmente independientes. Durante su primer llenado, sometido a un régimen temporal, sólo es colonizado por tres especies: Culiseta longereolata, Chironomus riparius y Dasyhelea sp.1. La progresiva disminución del volumen de agua y su perdida total, a principios de Abril, impide el asentamiento de otras especies, características de estos meses. Cuando se vuelve a llenar este estanque en Agosto, y se somete a un régimen de agua permanente, al igual que el resto de estanques, se observa una rapidísima colonización (a los 14 días, ya se han establecido 7 especies), favorecida por las altas temperaturas y la proximidad de las fuentes de posibles colonizadores, que constituyen el resto de estanques, aunque también es colonizado por Cladotanytarsus mancus que no está presente en los estanques.

El estanque X es colonizado por una sola especie Ephydra sp., que hace su aparición a principios de Junio y se mantiene hasta finales de Octubre.

\section{Variación temporal del espectro de abundancias relativas}

Del mismo modo que la importancia relativa de las especies varía entre estanques, sus esquemas de abundancia cambian también con el tiempo. Muchos organismos de vida corta, como la mayoría de las especies de insectos acuáticos registradas en los estanques, tienen una importancia relativa en la comunidad, que varía con la época del año, debido a que los individuos realizan su ciclo de vida de acuerdo con los cambios estacionales (BEGON et al., 1988).

En la Fig. 2 aparece representada para los nueve primeros estanques la variación temporal de la abundancia relativa de los diferentes órdenes de insectos acuáticos registrados que tienen

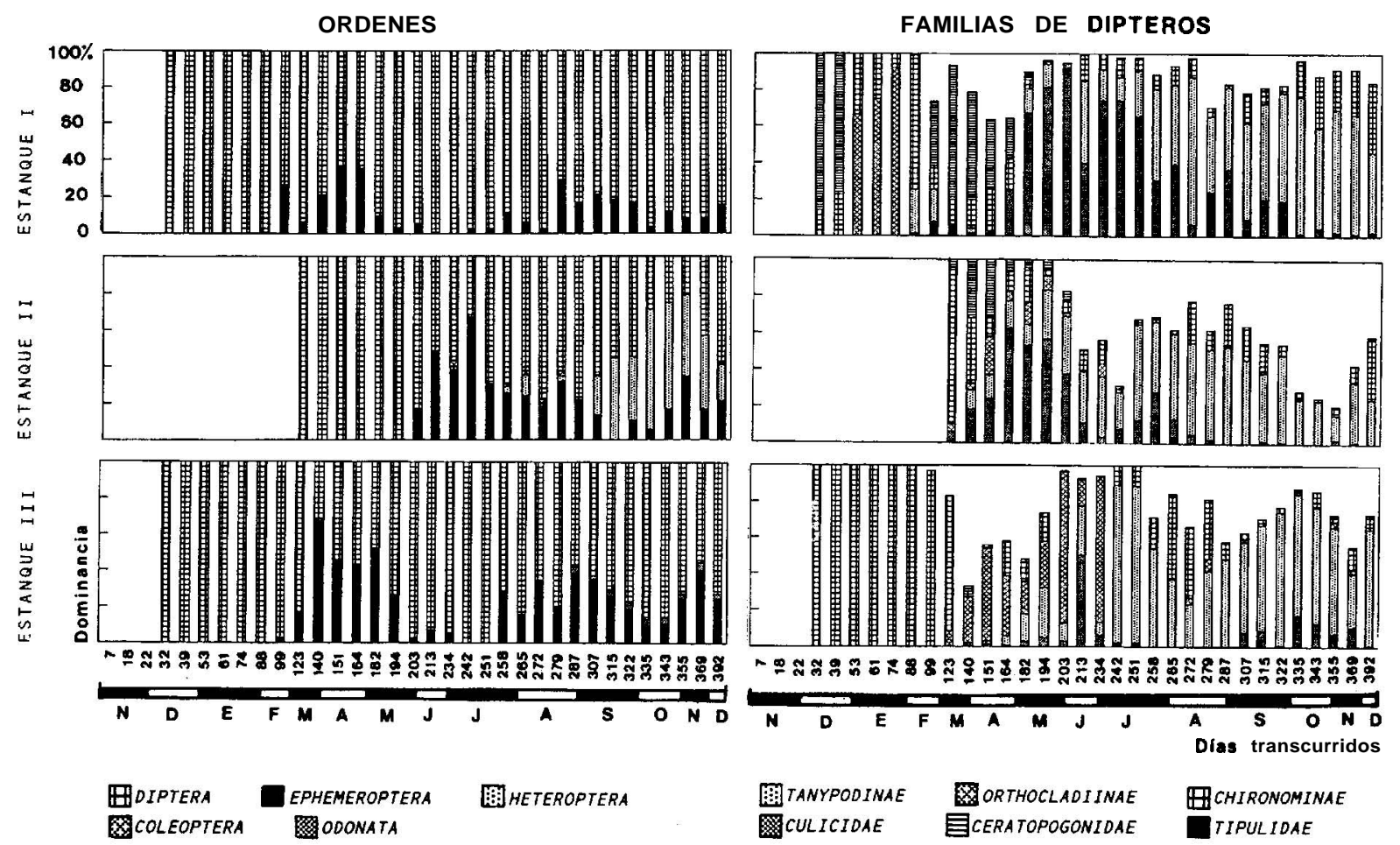

FIGURA 2: Variación temporal de las abundancias relativas de los diferentes ordenes de insectos acuáticos encontrados y de las familias de Dípteros (los Quironómidos son tratados a nivel de subfamilia).

FIGURE 2: Temporal variation in relative abundances of the different orders of aquatic insects found and of the Diptera families (Chironomides are treated to subfamily level). 

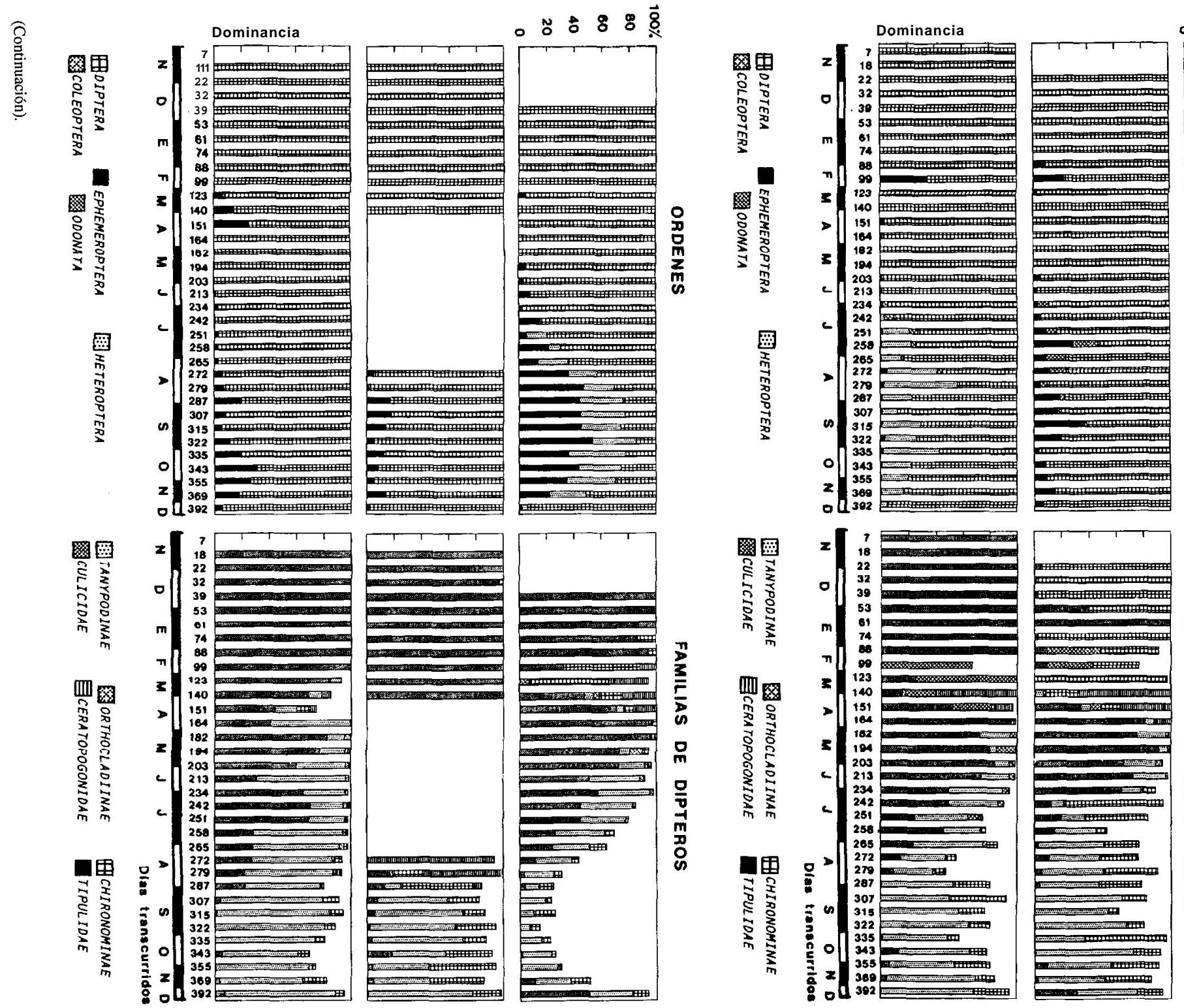

ก: $8: 8$
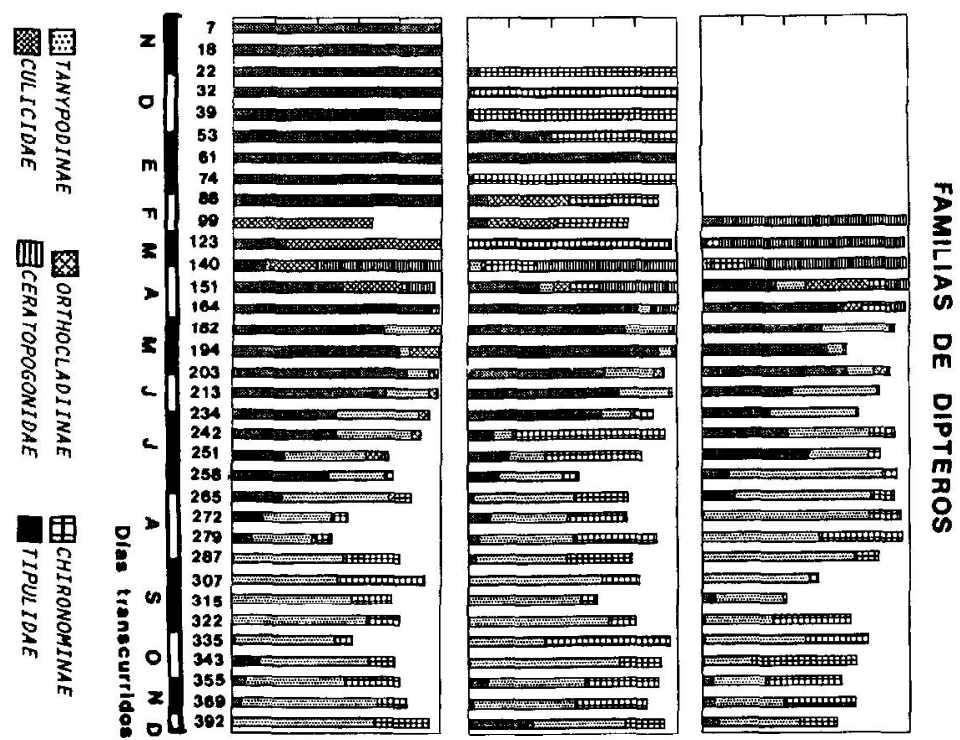
una importancia relativa superior al 1\%. Para los Dípteros, por ser el orden dominante, también se representa por familias, aunque para los Quironómidos, dada la gran variedad de tipos funcionales que presentan, se ha utilizado el nivel taxonómico de subfamilia.

Los Dípteros constituyen el orden de insectos dominante durante la mayor parte del periodo de estudio en todos los estanques, siguiéndole en importancia, los Efemerópteros, salvo en el estanque VI. Los Heterópteros llegan a ser una fracción importante de la comunidad en los estanques II, VI y VII. En cambio, Coleópteros y Odonatos representan una pequeña porción en los estanques donde se presentan.

Dentro de los Dípteros, los Culícidos, representados por Culiseta longeareolata, son dominantes en todos los estanques, excepto en el III, durante los meses de primavera, e incluso en los primeros meses, después del llenado, en los estanques VI, VIII y IX. Por el contrario, los Tanypodinos, representados por Procladius sagittalis, dominan durante los meses de verano y otoño, salvo en el estanque VII, donde la presencia de grava y la escasez de sedimento limita el desarrollo de larvas de Tanytarsinos, sus principales presas (BAKER \& McLACHLAN, 1979).

Los Quironominos, representados por Chironomus riparius durante la primera mitad del ciclo de estudio, no llegan a ser dominantes, salvo en los estanques III y V, donde alcanzan unas elevadas densidades de población, en los momentos iniciales de la colonización de estos medios, fundamentalmente en el estanque III. En la segunda mitad del ciclo, representados por Polypedilum laetum y varias especies de Tanytarsinos, adquieren el segundo papel en importancia, después de los Tanypodinos. Los mayores valores de abundancia los presentan en el estanque $\mathrm{V}$, debido al gran desarrollo de la población de Tanytarsus sp. 2 en este medio.

Los Orthocladinos, sólo llegan a ser dominantes en Enero, en el estanque I, en Febrero, en el estanque V, en Febrero y Marzo, en el estanque VI, y durante los meses de primavera en el estanque III. En el resto de estanques constituyen una fracción poco importante.

Los Ceratopogónidos, salvo en los estanques III, VIII (primer periodo) y IX, donde prácticamente son insignificantes, presentan una abundancia relativa elevada, normalmente, durante los meses de Febrero, Marzo y comienzos de Abril, e incluso, en el mes de Diciembre en el estanque I. En el estanque VIII, durante su segundo llenado, llegan a ser el grupo dominante en Agosto. Con respecto a los Tipúlidos, sólo constituyen una pequeña porción $(<10 \%)$ en el estanque I, durante los meses de Febrero y Marzo, cuando alcanzan su máximo desarrollo las poblaciones de Tipula sp.
Dentro de los Efemerópteros, la familia Baetidae, representada por Cloeon dipterwn, alcanza sus mayores porcentajes de abundancia, generalmente, durante la segunda mitad del periodo de estudio, aunque en los meses de primavera, en los estanques I y III, pueden llegar a superarlos. En ningún momento, salvo en una o dos fechas en los estanques II, III, IV y VII, esta especie domina con respecto a los Dípteros. La familia Caenidae, representada por Caenis luctuosa, tiene un porcentaje muy bajo en los estanques donde se presenta (II, III, IV y V).

Los Heterópteros, y dentro de estos los Coríxidos, constituyen una fracción importante, reflejo del gran desarrollo de las poblaciones de Heliocorisa vermiculata durante los meses de verano y otoño, en los estanques II y VII; y de Sigara lateralis en el estanque VI, en las mismas fechas. En algunas ocasiones este orden puede llegar a ser dominante frente a los Dípteros. En cambio, la familia Notonectidae, representa una pequeña porción en el estanque $\mathrm{V}$, donde Anisops debilis perplexa, desarrolla una única generación.

Con respecto a los Coleópteros, el Dytíscido Hydroglyphus pusillus, en los estanques IV, V y VI, y el Hydrofílido, Helochares lividus en el estanque II, son los únicos que tienen una abundancia relativa significativa, aunque no llegan a superar el $10 \%$, excepto Hydroglyphus pusillus en el estanque V, donde desarrolla varias generaciones durante los meses de verano.

En cuanto a los Odonatos, sólo llegan a alcanzar una cierta importancia las poblaciones de Ortethrum cancellatum en el estanque III, donde desarrolla su ciclo de vida a partir de Agosto.

Como se ha podido observar, sólo presentan una abundancia relativa importante en la comunidad, aquellas especies que desarrollan varios ciclos de vida en estos medios, y estas abundancias varían temporalmente en función, fundamentalmente, de los periodos reproductivos de las especies y sus requerimientos alimenticios.

\section{Variación temporal de la diversidad específica}

En la figura 3 se representa la variación temporal del índice de diversidad de Shannon- Wiener para los nueve primeros estanques. En general, en ninguno de los estanques, se observa una tendencia de incremento gradual de la diversidad, a lo largo del periodo de estudio, sino que estos fluctúan siguiendo un "pattem" más o menos estacional.

Durante los primeros meses de estudio, cuando los estanques sólo han sido colonizados por una o dos de las especies pioneras (Chironomus riparius y/o Culiseta longeareolata), se dan los valores de diversidad más bajos. En este periodo, y debido 


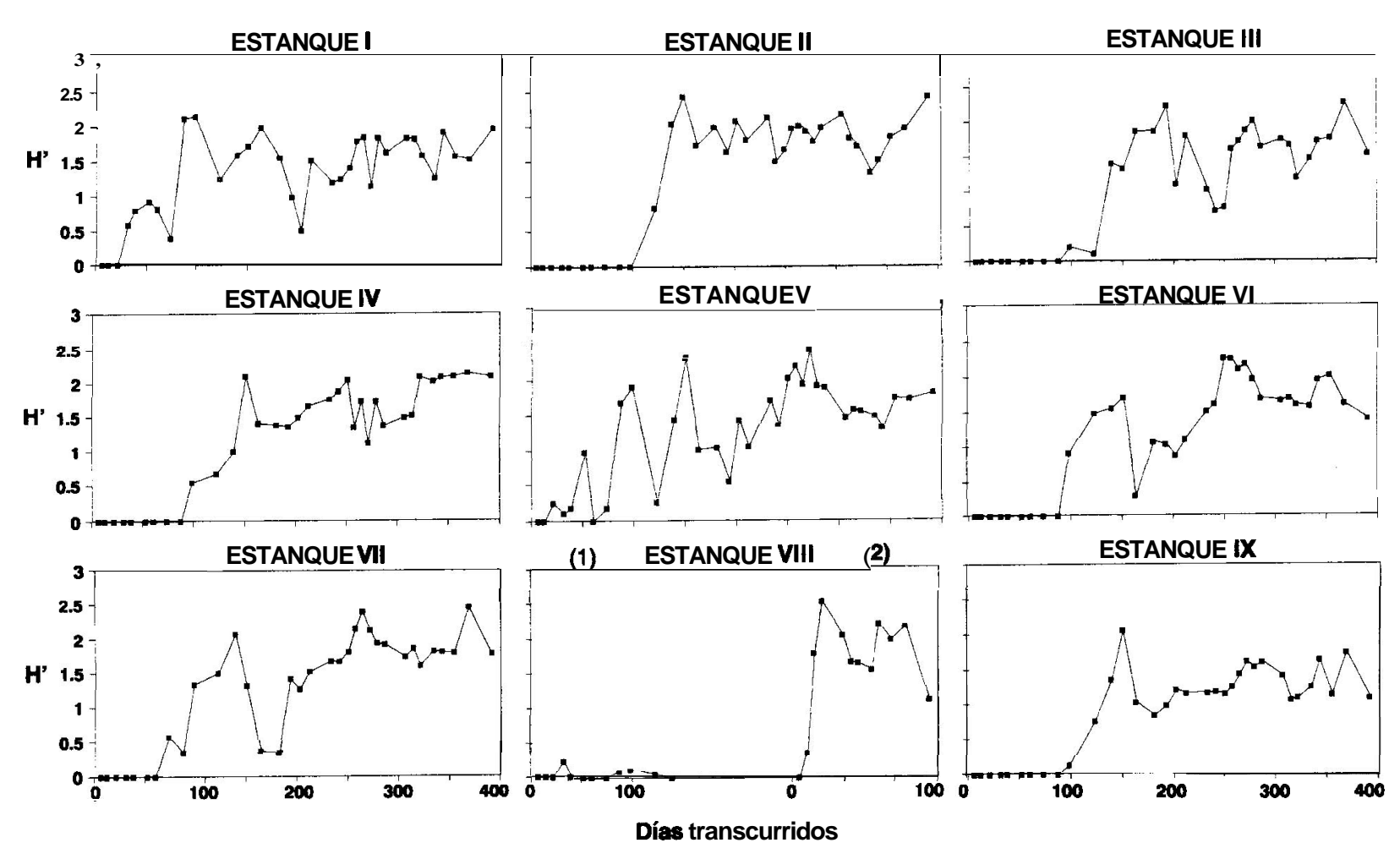

FIGURA 3: Variación temporal del índice de diversidad (Shanon-Wiener) para los nueve primeros estanques.

FIGURE 3: Temporal variation of Shannon-Wiener index for the first nine ponds.

a la gran cantidad de nichos ecológicos vacíos en estos medios nuevos, las relaciones inter e intraespecíficas de las poblaciones pioneras van a ser muy débiles.

En los meses siguientes, con el aumento gradual de la temperatura, que estimula la dispersión y reproducción de las especies de insectos acuáticos y el desarrollo de las comunidades de algas en los estanques, se establecen y desarrollan nuevas especies en estos medios, dando lugar a una comunidad más rica y compleja, con interacciones más intensas entre los organismos que la componen. Es en primavera, cuando se alcanzan generalmente los máximos valores de diversidad (ligeramente superiores a 2bits/individuo), reflejo del gran número de especies que se desarrollan en estas fechas en los estanques.

Posteriormente, en los primeros días de verano, se observa, normalmente, un declive de los valores de diversidad, resultado de la desaparición de determinadas especies típicas de primavera, como Dasyhelea sp.1, Chironomus riparius y algunos Orthocladinos, que tras completar sus ciclos de vida en los estanques, los abandonan; y de la disminución de las densidades de población de algunas especies que permanecen en estos medios, entre una generación y la siguiente.
En Julio y Agosto, vuelve a aumentar la complejidad de estos sistemas, con la llegada de los grandes depredadores, con ciclos de vida típicos de verano, como es el caso del Heteróptero Anisops debilis perplexa. La desaparición de estas poblaciones durante los meses de Septiembre y principios de Octubre, producen nuevamente una disminución de la diversidad de estos medios.

La mayoría de especies permanecen en otoño en forma larvaria, viéndose su desarrollo ralentizado con el descenso de temperaturas. Es en estas fechas cuando se observa un ligero aumento de los índices de diversidad.

Finalmente, cabe resaltar, que las mayores fluctuaciones del índice de diversidad, se producen en el estanque $\mathrm{V}$, durante la primavera, debido a los fuertes cambios en las densidades de población de sus especies dominantes (Culiseta longeareolata y Chironomus riparius).

\section{DISCUSIÓN}

Aunque la capacidad de un medio nuevo, para albergar colonizadores potenciales es grande, ya que están todos los nichos 
vacíos, las restricciones impuestas por el ciclo de vida de las especies, el comportamiento de los adultos (época y capacidad de dispersión, selección de los lugares de ovoposición, etc.), así como los requerimientos de las larvas, reducen en gran medida, el número de colonizadores con éxito.

La secuencia de llegada y establecimiento de las especies, aparte de estar determinada por las características propias de cada especie, depende de factores locales, como la acción de variables ambientales estresantes, la calidad y cantidad de alimento disponible y la presencia de especies competidoras $\mathrm{y} / \mathrm{o}$ depredadoras.

En el presente estudio los cambios atribuibles a la sucesión son muy difíciles de separar de aquellos relacionados con la estacionalidad. La estructura y organización de las comunidades parece tener un claro carácter estacional, determinado fundamentalmente por el régimen térmico. La temperatura marca tanto la estacionalidad de las especies como la del hábitat, ya que afecta directamente al desarrollo y crecimiento de los organismos, e indirectamente a la calidad y cantidad de alimento disponible, y a las características del ambiente fisico-químico donde se van a establecer las especies (VELASCO, 1989).

$\mathrm{Al}$ contrario de lo que ocurre en otras áreas templadas, donde la colonización por vuelo de insectos acuáticos está restringida a los periodos más calurosos del año, primavera y verano (WILLIAMS \& HYNES, 1976; WILLIAMS, 1980; VOSHELL \& SIMMONS, 1984), la colonización de los estanques, a pesar de haberse llenado al comienzo del periodo frío, es muy rápida, no viéndose limitada la llegada de especies, salvo en Enero, ocasionalmente, cuando se alcanzan temperaturas inferiores a $0^{\circ} \mathrm{C}$. Esta situación también ha sido observada en ríos temporales de California (ABELL, 1956).

A partir de la creación de estos medios, se va estructurando, con la llegada de especies invasoras, una comunidad biológica en perfecto ajuste con las variaciones sucesionales y estacionales del ambiente. En general, todos los estanques, excepto el VIII ( $1^{\text {er }}$ periodo) y X sometidos a la acción de factores ambientales estresantes (temporalidad y salinidad del agua, respectivamente), siguen una pauta de colonización y sucesión muy similar, distinguiéndose durante el primer año de su existencia diferentes etapas sucesionales que coinciden con los periodos estacionales.

Otros factores microambientales, como la presencia de macrófitos acuáticos, la escasez de sedimentos, el grado de insolación y la alta producción primaria por parte del fitoplancton, aunque no modifican la pauta general de colonización y sucesión, afectan a la selección del medio de las especies y a su establecimiento, determinando algunas diferencias en la composición y estructura de las comunidades de insectos acuáticos de los estanques.
La presencia de macrófitos acuáticos, en los estanques II y III, aumentan la heterogeneidad de estos medios y de microhábitats disponibles para las especies, ya que constituyen un excelente sustrato para la microflora y macroinvertebrados, a la vez que contribuyen considerablemente a la producción de detritos (DUDLEY, 1988). Esto se traduce en un aumento de la diversidad y equitabilidad de estos medios, con respecto al resto de los estanques (VELASCO et al. en este volumen). Sin embargo estos estanques son de los últimos en ser colonizados y donde se observan las densidades de población más bajas, fundamentalmente de Culícidos.

Algunas plantas inhiben la supervivencia de mosquitos inmaduros (FURLOW \& HAYS, 1972). CABALLERO (1919) y PARDO (1923), hacen algunas observaciones sobre las propiedades larvicidas de algunas especies de Caráceas. ANGERILLI \& BEIRNE (1984), también observan que las poblaciones de Culícidos son bajas en charcas que tienen vegetación acuática y piensan que puede ser debido a que la vegetación influye en la selección , por parte de las hembras, de hábitats para ovopositar, al producir cambios en la temperatura del agua y en su composición fisico-química, afectar la calidad y cantidad de materia particulada, así como a la visibilidad de la superficie del agua, a la vez que favorece la prescncia de especies depredadoras. En el presente estudio las dos especies de macrófitos, Chara vulgaris y Cladophora sp., tienen un efecto negativo sobre las poblaciones de Culiseta longeareolata, como era de esperar (HUTCHINSON, 1981), que parece afectar más al desarrollo de las larvas, que a la ovoposición de la especie, aunque en el estanque III también se observó una clara disminución en el número de puestas (VELASCO, 1989).

Por otra parte, la producción de ácidos químicos de Cladophora sp. impide el ramoneo de especies exclusivamente herbívoras (LALONDE et al., 1979), por lo que sus poblaciones, fundamentalmente Orthocladinos, se ven limitadas en el estanque II. En cambio, en el estanque III, Chara vulgaris, favorece la colonización y desarrollo de Haliplus lineatocollis, que se alimenta exclusivamente de ella, y de otras especies herbívoras, principalmente Orthocladinos, ya que constituye para ellos un excelente sustrato, donde refugio y alimento están asegurados (CASAS \& VÍLCHEZ, 1986).

La adición de nutrientes en el estanque $\mathrm{V}$, que desencadena un rápido e intenso desarrollo de fitoplancton durante los primeros meses del estudio, no parece acelerar el proceso de colonización, como cabía esperar, aunque si le permite mantener una comunidad más rica en individuos, pero no en especies, y donde sus poblaciones alcanzan las máximas densidades (VELASCO et al., en este volumen). La alta producción secundaria de este medio está estrechamente correlacionada con su producción primaria. 
El tipo de sustrato es considerado como uno de los factores más importantes en los primeros momentos de la colonización (PATERSON \& FERNANDO, 1969). Este hecho se constata en el presente estudio, sobre todo en las especies de Quironómidos. La escasez de sedimentos en los estanques VI y VII al inicio del estudio, retarda el establecimiento de especies típicamente detritívoras y limita, en parte, el desarrollo, sobre todo, de Quironómidos tubícolas.

El diferente grado de insolación que reciben los estanques, según un orden creciente, también parece afectar la colonización de estos medios. El estanque I, que es el que menor número de horas de sol recibe debido a su ubicación, y en principio el menos visible para las especies inmigrantes, presenta un número de especies sensiblemente menor (15) al del estanque IX (20), que es el de mayor exposición al sol. McLACHLAN (1988), encuentra una distribución diferente, entre dos especies de Quironómidos, en base a que las hembras discriminan las charcas, como lugares de ovoposición, por el grado de exposición al sol, apareciendo una de ellas en charcas umbrías y predominando la otra en charcas soleadas. Este autor manifiesta, que tal tipo de selección es común entre mosquitos y anfibios habitantes de charcas de lluvia. En el presente estudio, Tipula sp, también parece seleccionar los ambientes umbríos, ya que sólo coloniza los estanques I y II.

A todos estos factores macro y microambientales, así como los intrínsecos de las especies, que determinan los procesos de colonización general y el establecimiento de las especies, hay que añadir, a parte del factor azar, las interacciones biológicas, aunque en este trabajo no se han estudiado con detalle, ya que son difíciles de analizar y requieren evidencia experimental (McAULIFFE, 1983; PECKARSKY, 1983). Se han observado algunos hechos que pueden ser atribuibles a la competencia interespecífica entre especies afines taxonómica y ecologicamente: Chironomus riparius y Polypedilum laetum se suceden en el tiempo, Heliocorisa vermiculata y Sigara lateralis se excluyen en el espacio, aunque en otros casos, como Culiseta longeareolata y Culex pipiens pipiens, coexisten bajo condiciones de abundancia de alimento, pero predominando la primera de ellas sobre la segunda. Estas interacciones entre organismos, pueden ser vistas dentro del contexto sucesional según los mecanismos propuestos por CONNELL \& SLATYER (1977). La facilitación puede ser ilustrada por la secuencia desde los más tempranos colonizadores hasta los más tardíos. El hecho de que una especie llegue antes a un estanque e impida el asentamiento de otra con igual nicho ecológico es un claro ejemplo de inhibición; mientras que la coexistencia de las especies en un momento dado, ejemplifica la tolerancia.
Aunque es evidente, que en los medios estudiados, tanto factores abióticos como bióticos determinan los procesos de colonización y sucesión, la importancia relativa de cada uno de ellos no lo es tanto. Sólo cuando el medio está sometido a la acción de un factor ambiental estresante, como la alta salinidad del agua o la temporalidad del medio, son estos factores estresantes los principales determinantes.

\section{AGRADECIMIENTOS}

Agradecemos a $\mathrm{M}^{\mathrm{a}}$.R. Vidal-Abarca y $\mathrm{M}^{\mathrm{a}}$.L. Suárez Alonso sus comentarios y sugerencias.

\section{BIBLIOGRAFÍA}

ABELL, D.L. 1956. An ecological study of intermittency in foothill streams of Central California. Ph. D. Thesis. Univ. Califomia. Berkeley.

ANGERILLI. N.P.D. \& BEIRNE, B.P. 1984. Influences of aquatic plants on colonization of artificial ponds by mosquitoes and their insects predators. Can. Ent., 112 : 793-796.

BAKER, A.A. \& McLACHLAN, A.J. 1979. Food preferences of Tanypodinae larvae (Diptera: Chironomidae). Hydrobiologia, 62: 283-288.

BEGON, M; HARPER, J.L. \& TOWNSEND, L.C. 1988 Ecología. Individuos, poblaciones y comunidades. Ed. Omega. Barcelona. 886 pp.

CABALlERO, A. 1919. La Chara faetida A. Br. y las larvas de Stegeomyia, Culex y Anopheles. Bol. Roy. Soc. Esp. Hist. Nat. Madrid 19(8): 449.

CASAS, J.J. \& VÍLCHEZ, A. 1986. Factores que afectan a la distribución de las larvas de Quironómidos en las aguas de los estanques de la Alhambra y el Generalife. Limnética, 2: 63-74.

CONNELL, J.P. \& SLATYER, R.O. 1977. Mechanisms of succession in natural communities and their role in community stability and organization. Amer. Nat., 111: 1119-1144.

CONNELL, J.P.; NOBLE, I.R. \& SLATYER, R.O. 1987. On the mechanisms producing successional change. Oikos, 50(1): 136-137.

DUDLEY, T.L. 1988. The roles of plant complexity and epiphyton in colonization of macrofites by stream insects. Verh. Internat. Verein. Limnol., 23 : 1153-1158.

FISHER, S.G. 1983. Sucession in Streams. In: Application and Testing of General Ecological Theory. (BARNES, J.R. \& MINSHALL, G.W. eds.): 7-27. Plenwn Press. New York.

FRANCISCOLO, M.E. 1979. Fauna d'Italia. Coleoptera: Haliplidae, Hygrobiidae, Gyrinidae, Dytiscidae. Edizioni Calderini. Bologna. 804 pp. 
FURLOW, B.M. \& HAYS, K.L. 1972. Some influence of aquatic vegetation on the species and number of Culicidae (Diptera) in small pools of water. Mosquito News, 32(4): 595-599.

HUTCHINSON, G.E. 1981. Thoughts on aquatic insects. BioScience, 31: 495-500.

LALONDE, R.T. et al. 1979. Response of Aedes seriatus larvae to fatty acids of Cladophora. J. Chem. Ecol. 5:371-381.

MALMQVIST, B.; RUNDLE, S.; BRONMARK, C. \& ERLANDSSON, A. 1991. Invertebrate colonization of a new, man-made strearn in southern Sweden. Freshwater Biology, 26: 307-324.

McAULIFFE, J.R. 1983. Competition, colonization patterns, aand disturbance in stream benthic communities. In: Stream ecology: Aplication and Testing of General Ecologycal Theory. (BARNES, J.R. \& MINSHALL, G.W. eds.): 137-156. Plenum Press. New York.

McLACHLAN, A.J. 1988. Refugia and habitat partitioning amog midges (Diptera: Chironomidae) in rain-pools. Ecological Entomology, 13 : 185-193.

PARDO, L. 1923. Observaciones acerca de la acción de la Chara sobre las larvas de los mosquitos. Bol. Soc. Esp. de Hist. Nat. Tomo XXIII
PINDER, L.C.V. 1986. Biology of freshwater Chironomidae. Ann. Rev. Entomol., 31: 1-23.

TANSLEY, A.G. 1929. Succession: the concept and its value. Internat. Congr. Plant. Sci., Ithaca, Proc. 1926. 1: 677-686.

VELASCO, J. 1989. Estudio de los procesos de colonización de medios artificiales por insectos acuáticos en el Sureste Iberico. Tesis Doctoral, Universidad de Murcia, 502 pp.

VELASCO, J.; MILLÁN, A.; \& RAMÍREZ-DÍAZ, L., en este volumen. Colonización y sucesión de nuevos medios acuáticos. I. Composición y estructura de las comunidades de insectos. Limnética, 9: 73-85.

VOSHELL, J.R. \& SIMMONS, G.M. 1984. Colonization and sucession of benthic macroinvertebrates in a new reservoir. Hydrobiologia, 112: 27-39.

WILLIAMS, D.D. 1980. Temporal patterns in recolonization of stream benthos. Archiv für Hydrobiologia, 90: 56-74.

WILLIAMS, D.D. \& HYNES, H.B.N. 1976. The Ecology of Temporary Streams. I. The Faunas of Two Canadian Streams. Int. Revue ges. Hydrobiol, 61 (6): 761-787. 